\title{
Non-Invasive Multi-Scale Imaging and Modelling Using X-Ray Microscopy
}

Matthew Andrew ${ }^{1}$, Renata Chica-Szot ${ }^{2}$, Sven Liden ${ }^{3}$, Piotr $\mathrm{Such}^{2}$, Grzegorz Lesniak ${ }^{2}$, Andreas Wiegmann ${ }^{3}$, Jeff Gelb ${ }^{1}$, Mike Marsh ${ }^{4}$, Andy Steinbach ${ }^{1}$

1. Carl Zeiss X-ray Microscopy, Pleasanton, CA, USA

2. INIG, National Research Institute, ul. Lubicz 25A, 31-503 Kraków, Poland

3. Math2Market, Stiftsplatz 5, 67655 Kaiserslautern, Germany

4. Object Research Systems, Montreal, Canada

Over the last 10-15 years 3 dimensional x-ray microscopy (XRM) has emerged as the leading technology for the non-invasive imaging of materials ranging from the multi-cm to the sub-micron scales. One of the principal challenges when implementing such technology is how to deal with multiscale heterogeneity. Frequently the high resolutions required to capture the fundamental length scales governing physical phenomena come at the sacrifice of a field of view required to capture macroscopic heterogeneity. Multi-scale methods and techniques are required to bridge this gap, allowing for integrated classification, sampling and characterization workflows to be developed. While methodological techniques have been developed which allow for multiple imaging scales and modalities to be integrated, they tend to require significant careful manual interaction for the required image site location and subsequent image registration (e.g. [1]) limiting the utility and applicability of the resulting technique. X-ray microscopy offers unique advantages in solving such a challenge, as the variety of detectors integrated into a single imaging system allows for multiple scales to be proscriptively imaged with minimal user interaction. In this paper we present a novel methodology for integrating multi-scale, non-invasive 3D acquisition and analysis, applied to a heterogenous subsurface sandstone sample using the ZEISS Versa XRM 520 and ORS Visual SI Advanced software. Geological materials provide a unique test for such workflows, as they exhibit strong heterogeneity at all length scales [2].

First the entire 1" $(25 \mathrm{~mm})$ core-plug sample was imaged across its entire volume with a resolution of around $19 \mu \mathrm{m}$. While the detailed pore structure was not resolvable at this resolution, the macroscopic heterogeneity associated with the strong sedimentary layering was, along with a prominent crack through the entire volume of the sample. This image was then classified using advanced rock typing techniques such that the greyscale image was converted into a label image where each voxel was either labelled as a "high porosity lithology", "low porosity lithology" or belonging to the crack. By the automated incorporation of spatial meta-data from a high accuracy micro-positioning stage mounted on top of the rotational axis required for tomographic imaging, it is possible to directly locate positions for interior tomography directly from a $3 \mathrm{D}$ representation of the reconstructed data (figure 1). It was therefore possible to define locations for interior tomography with a positional accuracy of around $1 \mu \mathrm{m}$. This allows for proscriptive multi-scale 3D image sampling with an accuracy never before possible at arbitrary positions within a macroscopic volume.

The high resolution interior tomographies were used as inputs for the commercially available GeoDict pore-scale modelling software and the permeability tensors were calculated[3]. As high resolution positional association was maintained, the relationship between the macroscopic lithological classification of rock type and microscopic pore-scale prediction could be maintained. This allowed for the construction of a macroscopic core-plug scale model based on the low resolution classified image, such that each voxel from the high porosity lithology and the low porosity lithology was assigned the 
associated permeability tensor derived from their associated high resolution images. A multi-scale simulation was then performed where stokes flow was computed through the macroscopically poreresolved fracture and Darcy flow was calculated for voxels in regions where sampled lithologies were assigned permeabilities derived from the higher resolution scans and modelling. (figure 2). The resulting permeability tensor was highly anisotropic, with a high permeability in directions parallel to layering and low permeabilities perpendicular to it. This result was only possible because the $\mathrm{cm}$ scale heterogeneity associated with primary sedimentary layering was maintained, through the use of the macroscopic model.

References:

[1] A. Merkle, L. L. Lavery, J. Gelb, and N. Piché, Microsc. Microanal., vol. 20, no. Supplement S3, pp. 820-821,2014.

[2] P. S. Ringrose, a. W. Martinius, and J. Alvestad, Geol. Soc. London, Spec. Publ., vol. 309, no. 1, pp. 123-134,2008.

[3] S. Linden, A. Wiegmann, and H. Hagen, Graph. Models, vol. 82, pp. 58-66, 2015.

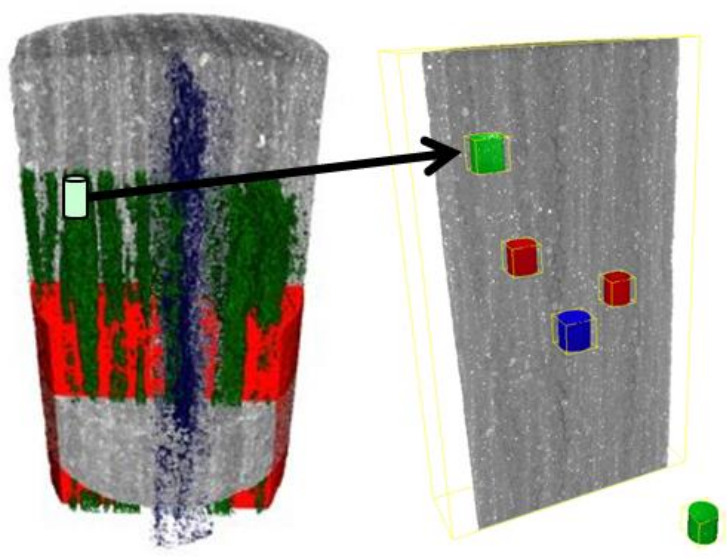

Figure 1. Macroscopic classification and sampling of a heterogeneous sandstone showing high porosity (green), low porosity (red) and crack (blue) lithologies. Resulting interior tomographic positions shown with associated colours on the right.

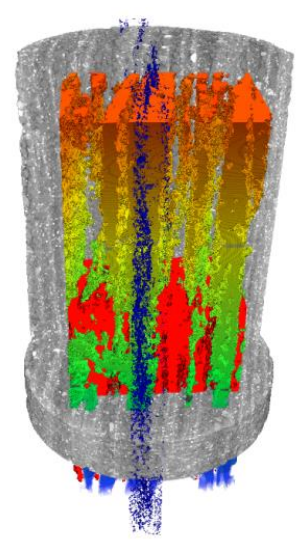

Figure 2. Up-scaled model constructed using macroscopic classification image, showing prominent permeability anisotropy as a result of $\mathrm{cm}$ scale heterogeneity. 